\title{
O USO INADEQUADO DE CLORIDRATO DE METILFENIDATO POR ESTUDANTES COM INTUITO DE AUMENTAR DESEMPENHO COGNITIVO
}

\author{
THE INAPPROPRIATE USE OF METHYLPHENIDATE HYDROCHLORIDE BY \\ STUDENTS IN ORDER TO INCREASE THEIR COGNITIVE PERFORMANCE
}

\author{
Jéssica de Oliveira Sampaio Antunes ${ }^{1}$ \\ Fernando Augusto de Freitas ${ }^{2}$ \\ Rafaela Dal Piva ${ }^{3}$
}

RESUMO: O cloridrato de metilfenidato conhecido popularmente como Ritalina ${ }^{\circledR}$, é um estimulante do sistema nervoso central (SNC) e uma das finalidades do deste medicamento é para o tratamento de Transtorno do Déficit de Atenção com Hiperatividade (TDAH) e narcolepsia, mas atualmente o seu uso se estende ao consumo indiscriminado deste medicamento por pessoas que procuram melhorar o desempenho em concursos e situações afins. Sendo assim, deste trabalho consistem em averiguar os riscos e a extensão do uso indiscriminado do metilfenidato por estudantes universitários que não possuem um diagnóstico de TDAH, mas que se automedicam buscando um rendimento cognitivo nos estudos, bem como diagnosticar quais são as principais motivações de tal prática. Foi realizada uma revisão bibliográfica dos artigos cientificos do tipo narrativa, sendo que coleta dos dados ocorreu nas bases de dados bibliográficos PubMed, SciELO e Google Acadêmico. No processo para inclusão, foram considerados os artigos científicos em suporte eletrônico ou impressos, com o texto completo em Português ou Inglês, bem como informações disponibilizadas em endereços eletrônicos de agências e órgãos oficiais. Os resultados das pesquisas descritas neste trabalho demonstram que o consumo entre estudantes de universidades brasileiras está compatível com estudos internacionais similares, que indicam que o uso está dentro de uma faixa de $1,5 \%$ a $31 \%$ dos envolvidos nas pesquisas. Como exemplo, em um estudo realizado na Faculdade Anhanguera de Brasília com 400 alunos, 5,8\% afirmaram que já haviam feito uso do

${ }^{1}$ Graduanda do Curso de Farmácia do Centro de Ensino Superior de Foz do Iguaçu (CESUFOZ), jessicasampaiooo8@gmail.com.

${ }^{2}$ Docente do curso de Farmácia do Centro de Ensino Superior de Foz do Iguaçu (CESUFOZ),

fernandoaugustodefreitas.faf@gmail.com.

3Orientadora: Docente do curso de Farmácia do Centro de Ensino Superior de Foz do Iguaçu (CESUFOZ), rafaeladalpiva@yahoo.com.br. 
metilfenidato em algum momento, enquanto outro estudo com estudantes de Medicina da Universidade Federal da Bahia, mostrou que 8,6\% dos entrevistados afirmaram que já haviam utilizado o metilfenidato em algum momento da vida escolar. Sendo assim, considerando que a utilização indiscriminada de metilfenidato, bem como outros psicoestimulantes, pode ocasionar efeitos colaterais imediatos ou a longo prazo, torna-se necessário que haja um maior controle da sua prescrição e dispensação, bem como uma atenção especial por parte das autoridades responsáveis para uma maior conscientização dos alunos a respeito dos riscos inerentes ao seu uso indiscriminado.

Palavras-chave: Metilfenidato. Auto-prescrição. Transtorno de déficit de atenção e hiperatividade.

ABSTRACT: Methylphenidate hydrochloride, popularly known as Ritalin ${ }^{\circledR}$, is one Central Nervous System (CNS) stimulant. One of the purposes of this medication is to treat Attention Deficit Hyperactivity Disorder (ADHD) and narcolepsy. However, currently, its use extends the indiscriminate consumption of this drug by people seeking to improve performance in competitions and similar situations. Thus, this work investigates the risks and extent of the non-medical use of methylphenidate among undergraduate students who do not possess any disorder to enhance their cognitive functions and diagnose the primary motivations for such practice. In this article, a literature review of the narrative type was carried out, and data collection took place in the bibliographic databases PubMed, SciELO, and Academic Google. In the inclusion process, scientific articles in electronic or printed support were considered, with the full text in Portuguese or English and information available on the electronic addresses of agencies and official bodies. The data analysis described in this paper demonstrates that consumption among Brazilian university students is compatible with similar international studies, indicating that use is within $1.5 \%$ to $31 \%$ of those involved in the surveys. As an example, in a study carried out at Anhanguera School of Brasilia with 400 students, $5.8 \%$ said they had already used methylphenidate at some point. In addition, another study with medical students at the Federal University of Bahia showed that $8.6 \%$ of respondents stated that they had already used methylphenidate at some point in their school life. Therefore, considering that the indiscriminate use of methylphenidate, as well as other psychostimulants, can cause immediate or long-term side effects, it is necessary to have greater control over its prescription and dispensing, as well as special attention by the authorities responsible for greater awareness of students about the risks inherent in its indiscriminate use.

Keywords: Methylphenidate. Self-prescription. Attention Deficit Hyperactivity Disorder. 


\section{INTRODUÇÃO}

O cloridrato de metilfenidato conhecido popularmente como Ritalina ${ }^{\circledR}$, é um estimulante do sistema nervoso central (SNC) e faz parte da lista A3 da Portaria 344, de 12 de maio de 1998, que o inclui na lista das substâncias psicotrópicas (sujeitas a Notificação de Receita "A") (ANVISA, 2020). O cloridrato de metilfenidato (CM) começou a ser vendido nos anos 50 na Suíça, Alemanha e nos Estados Unidos, mas teve o seu comércio intensificado quando foi associado ao tratamento do Transtorno do Déficit de Atenção e Hiperatividade (TDAH) (ITABORAHY; ORTEGA, 2013). Atualmente diversos fatores contribuem para o aumento do consumo de fármacos psicoestimulantes e, entre eles, destacam-se a expansão das indústrias farmacêuticas e o acesso facilitado da população aos medicamentos, visando o progresso nas atividades intelectuais e também para finalidades recreativas (ROCHA et al., 2020).

Os estimulantes do Sistema Nervoso Central (SNC) aumentam o estado de vigília e levam a uma situação de alerta, com a consequente melhora do humor e desempenho cognitivo (SANTANA et al., 2020). Uma das finalidades do uso de metilfenidato é para o tratamento TDAH, com intuito de desenvolvimento mental e concentração do indivíduo nos estudos e no seu âmbito profissional, mostrando uma melhora considerável nos sintomas da desatenção. Porém, o efeito real obtido em pessoas saudáveis que fazem uso desse medicamento é questionado pela literatura científica por falta de comprovações (MONTEIRO et al., 2017).

Diversos estudos demonstram um alto consumo de metilfenidato relacionado com pessoas que procuram melhorar o desempenho em concursos e situações afins, mesmo não havendo recomendações clínicas para o uso do fármaco, o que tem levado ao consumo indiscriminado do metilfenidato (COLI; SOUSA E SILVA; NAKASU, 2016), o que é corroborado pelos dados mostrados no Fórum sobre Medicalização da Educação e da Sociedade a partir de dados do Sistema Nacional de Gerenciamento de Produtos Controlados ANVISA (2007-2014). Em tal estudo, verificou-se um aumento de $180 \%$ em 4 anos no 
consumo do medicamento Ritalina ${ }^{\circledR}$, passando de 58.719 caixas vendidas em outubro de 2009, para 108.609 caixas em outubro de 2013 (HARAYAMA et al., 2015).

Os efeitos adversos mais comuns estão relacionados com aumento do nervosismo, insônia, diminuição de apetite, ansiedade e agitação. Portanto, o uso disseminado causa vício e danos colaterais por meses, mesmo após ser interrompido o uso do fármaco, assim trazendo risco para saúde dos acadêmicos quando usado de forma irresponsável (MOTA; PESSANHA, 2014). Sendo assim, os objetivos desta revisão de literatura narrativa consistem em averiguar os riscos e a extensão do uso indiscriminado do metilfenidato por estudantes universitários que não possuem um diagnóstico de $\mathrm{TDAH}$, mas que se automedicam buscando um rendimento cognitivo nos estudos, bem como diagnosticar quais são as principais motivações de tal prática.

\section{JUSTIFICATIVA}

O ambiente acadêmico impõe uma grande pressão pelo alto rendimento nos processos de avaliação para os mais variados processos seletivos, o que torna tais estudantes os principais usuários do uso indiscriminado do cloridrato de metilfenidato. Como justificativa para o consumo, os usuários alegam obter melhoras nos níveis de concentração e não levam em consideração os possíveis efeitos adversos que podem aparecer a longo prazo. Tendo em vista a necessidade de prescrição médica para a obtenção do metilfenidato, diversas estratégias são utilizadas pelos usuários para a contornar tal dificuldade, como o desvio de medicamentos obtidos regularmente por parentes, simulação de sintomas durante atendimentos médicos, bem como prescrições fraudulentas (MONTEIRO et al., 2017).

Assim, o presente trabalho se justifica por avaliar a extensão do uso do metilfenidato no ambiente universitário em diferentes localidades brasileiras, baseando-se em estudos de casos já publicados, o que ocorre mesmo com a ausência de dados que corroborem possíveis efeitos benéficos no uso da substância e, além disso, sem que sejam considerados os possíveis efeitos colaterais, como insônia, cefaleia, redução do apetite e perda de peso, entre outros. 


\section{REVISÃO TEÓRICA}

O metilfenidato (também encontrado sob a forma de um sal) é derivado da piperidina, uma amina heterocíclica com um anel de seis membros contendo cinco átomos de carbono e um átomo de nitrogênio, e da fenetilamina comum à dopamina e noradrenalina. $\mathrm{O}$ nome químico é metil 2-fenil-2-(2-piperidil) acetato e sua fórmula molecular é $\mathrm{C}_{\mathrm{I} 4} \mathrm{H}_{\mathrm{I} 9} \mathrm{NO}_{2}$ (FREESE et al., 2012), como mostrado na Figura I.

Figura I. Estrutura do metilfenidato.

Fonte: Baseado em NLM (2004).

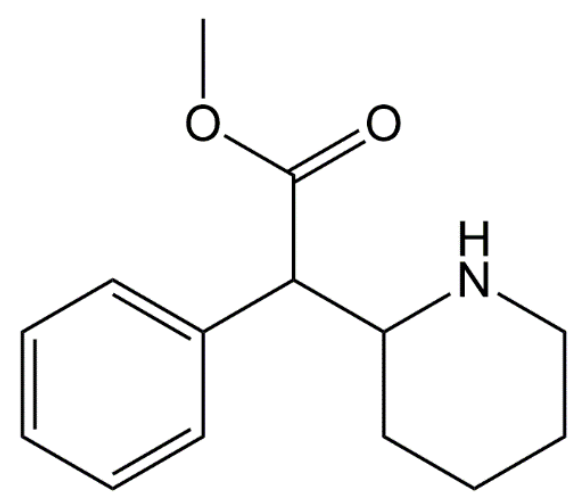

O metilfenidato também pode ser encontrado em sua forma salina (cloridrato de metilfenidato) e é comercializado como a mistura racêmica de (d)-metilfenidato e (1)metilfenidato, sendo que estudos demonstram que a molécula (d)-metilfenidato é a responsável pela ação farmacológica do medicamento, bem como pelos efeitos adversos associados ao seu uso (FREESE et al., 2012).

No sistema nervoso central (SNC), os neurotransmissores dopamina e noradrenalina são liberados no córtex pré-frontal e estimulam receptores específicos nos neurônios póssinápticos, mecanismo pelo qual ocorre a transmissão de sinais e descargas neuronais e, além disso, neurotransmissores específicos também são requeridos sob certas circunstâncias. Da mesma maneira que a especificidade do sinal depende do tipo de neurotransmissor liberado no espaço sináptico, as concentrações destas moléculas também definem o tipo de sinal a ser propagado (ANDRADE, 2018). 
Após a ação na transmissão de sinais nas sinapses, diferentes mecanismos promovem a rápida retirada no neurotransmissor das fendas, uma vez que existe uma necessidade de interrupção do sinal após a ação requerida. Alguns neurotransmissores são inativados após a transmissão do sinal, enquanto outros também podem ser capturados pelo neurônio présináptico ou outras células auxiliares (células da glia). A captura dos diferentes neurotransmissores é dependente da presença de proteínas transportadoras e, no caso da dopamina e noradrenalina, a captação ocorre por seus transportadores específicos aos neurônios dopaminérgicos e noradrenérgicos, respectivamente, sendo armazenados posteriormente em vesículas. Sendo assim, os transportadores de neurotransmissores monoaminados são alvos de diversas drogas psicoativas, como estimulantes, antidepressivos e ansiolíticos.

Da mesma forma que as anfetaminas, cloridrato de metilfenidato atua bloqueando os transportadores de dopamina e noradrenalina, o que impede a recaptação destes neurotransmissores pelo terminal pré-sináptico e leva ao aumento da disponibilidade sináptica de ambos. Sendo assim, o fármaco impede que as catecolaminas sejam recapturadas pelas terminações nervosas, fazendo com que ambos permaneçam ativos por mais tempo no espaço sináptico, promovendo um aumento da concentração, coordenação motora e excitação (ANDRADE et al., 2018). Entretanto, um aumento das concentrações de dopamina e noradrenalina provocado pelo uso indiscriminado de metilfenidato em indivíduos saudáveis pode desencadear vários efeitos adversos, o que pode incluir até euforia, delírio, confusão, psicose tóxica e alucinações (MORTON; STOCKTON, 2000).

O cloridrato de metilfenidato é usado para tratamento desde crianças a adultos com déficit de atenção e hiperatividade (TDAH), conhecida como síndrome hipercinética, os quais podem apresentar, entre outros sintomas, dificuldades de concentração em atividades de longa duração, excitabilidade, esquecimento ou falta de atenção, ansiedade, depressão ou dificuldade de aprendizagem (SANTOS; VASCONCELOS, 2010). De acordo com Associação Brasileira de Déficit de Atenção (ABDA) o TDAH pode ser considerado um transtorno neurobiológico 
que pode acometer o indivíduo desde a infância até a idade adulta, sendo predominante em pessoas do sexo masculino (ROSA; TELLES, 2009).

Apesar dos reconhecidos benefícios obtidos pelo uso do cloridrato de metilfenidato no tratamento do TDAH em crianças e adultos, bem como da narcolepsia, diversos estudos relatam o uso indiscrimidado do medicamento. Como consequência dessa tendência, no ano de 2010 verificou-se um aumento na produção mundial de metilfenidato, com um recorde de 43 toneladas, o que representou um número 20 vezes mais alto do que a produção registrada na década de 90 , além de corresponder a aproximadamente $56 \%$ do volume total dos estimulantes produzidos no mundo. Os EUA são os maiores consumidores desse psicoestimulante e, entre o período de 1990 a 2005, verificou-se um aumento de seis vezes em sua produção (DOMITROVIC; CALIMAN, 2018). O aumento do consumo também foi observado no Brasil, conforme dados do Conselho Regional de Farmácia do estado de São Paulo, onde consta que foram consumidos nacionalmente $94 \mathrm{~kg}$ do medicamento em 2003 e $875 \mathrm{~kg}$ em 2012, com um crescimento observado de $775 \%$ (CRF-SP, 2014).

\section{METOdOLOGIA}

Foi realizada uma revisão bibliográfica dos artigos cientificos do tipo narrativa, onde o levantamento do material para o presente estudo tiveram como base os descritores ou palavras-chave "cloridrato de metilfenidato", "riscos do cloridrato de metilfenidato à saúde", "tratamento de TDAH", "cloridrato de metilfenidato e seu uso nas universidades" e “mecanismo de ação do cloridrato de metilfenidato". A coleta dos dados ocorreu nas bases de dados bibliográficos PubMed (www.ncbi.nlm.nih.gov), SciELO (www.scielo.org) e Google Acadêmico (https://scholar.google.com.br). No processo para inclusão, foram considerados os artigos científicos em suporte eletrônico ou impressos, com o texto completo em Português ou Inglês, bem como informações disponibilizadas em endereços eletrônicos de agências e órgãos oficiais. 


\section{RESULTADOS E DISCUSSÃO}

O crescente uso indiscriminado do metilfenidato no meio acadêmico é uma tendência observada em diversos países, não só no Brasil, tendo em vista a pressão social alto rendimento nos processos de avaliação para os mais variados processos seletivos, entre outras situações. O conhecimento das características dos diferentes grupos etários, sociais e de formação acadêmica são de grande importância para direcionar as ações públicas de saúde que visam o esclarecimento da população, bem como o combate ao uso abusivo de estimulantes.

O livre acesso à internet e sua atual abrangência na população são características que levam a um ambiente propício para a aquisição de medicamentos de forma irregular, sem prescrição e acompanhamento médico, muitas vezes com informações equivocadas ou incompletas a respeito do assunto. A aquisição e uso de medicamentos sem a devida orientação, no entanto, não é influenciada apenas pela internet, já que diversos estudos demonstram que tais usuários obtém os medicamentos desejados por meio de parentes, orientação com amigos e até diretamente com farmacêuticos.

Diferentes estudos demonstram que as porcentagens de estudantes universitários que fazem uso indiscriminado do metilfenidato podem variar significativamente, o que pode ser explicado por vários motivos, sendo que o nível socioeconômico dos entrevistados e a área de formação, desempenham papel relevante nos resultados das pesquisas realizadas. De forma geral, no entanto, estima-se que o uso esteja dentro de uma faixa de $1,5 \%$ a $31 \%$ dos envolvidos nas pesquisas (BOGLE; SMITH, 2009).

Como exemplo, em um estudo realizado nos cursos de Biomedicina, Enfermagem, Farmácia e Nutrição da Faculdade Anhanguera de Brasília, 400 alunos responderam um formulário de pesquisa e 5,8\% $(n=23)$ afirmaram que já haviam feito uso do metilfenidato em algum momento, sendo que apenas $34,8 \%(n=8)$ deles possuíam prescrição médica. No mesmo estudo, os autores mostraram que 9\% obtiveram orientações para o uso em endereços eletrônicos na internet, sendo que o mesmo índice foi observado entre aqueles cuja fonte de informação foi diretamente com farmacêuticos. Finalmente, os $48 \%$ restantes dos usuários 
buscaram informações com amigos (AFFONSO et al., 2016). Outro estudo realizado entre estudantes de Medicina da Universidade Federal da Bahia, mostrou que 8,6\% dos entrevistados (de um total de 185 alunos) afirmaram que já haviam utilizado o metilfenidato em algum momento da vida escolar, tendo como objetivo a melhora no rendimento acadêmico (CRUZ et al., 20II).

Tais resultados estão dentro da faixa estimada de $1,5 \%$ a $31 \%$ citada anteriormente (BOGLE; SMITH, 2009), o que também é corroborado por outro estudo realizado com i6o estudantes do $\mathrm{I}^{\circ}$ ao $8^{\circ}$ período de Medicina do Centro Universitário de Volta Redonda, onde 23,72\% responderam que já haviam feito uso do metilfenidato sem prescrição médica (CARNEIRO et al., 2013). Da mesma maneira, os dados relativos a um estudo realizado com 200 estudantes de graduação em Medicina da Universidade Federal do Rio Grande (FURG), que avaliou o consumo de diversos psicoestimulantes e, entre eles o metilfenidato, mostrou que $5,5 \%(n=$ II $)$ dos alunos participantes consumiam tal medicamento no momento da pesquisa, sendo que não foi observada a prevalência significativa de nenhum sexo para o conjunto de todos os psicoestimulantes considerados (52,6\% para os rapazes e 50,9\% para as moças) (MORGAN et al., 2017).

O cenário observado nacionalmente com relação à porcentagem de alunos que utilizam indiscriminadamente o metilfenidato está coerente com estudos internacionais, como mostra uma pesquisa realizada entre estudantes da Massachusetts College of Liberal Arts (MCLA, EUA), no qual 283 alunos responderam o questionário aos pesquisadores e $16 \%$ afirmaram já terem feito uso do metilfenidato sem receita médica, para fins de melhora cognitiva (BABCOCK; BYRNE, 200o).

No entanto, tendo em vista a influência de aspectos socioeconômicos e das diferentes áreas de formação dos estudantes entrevistados, entre outros aspectos, existem estudos que apresentam resultados bastante discrepantes dos já citados, como é o caso de uma pesquisa realizada para avaliar a prevalência do uso de metilfenidato por universitários dos cursos de Farmácia e Medicina na cidade de Campos dos Goytacazes/RJ. Nesse estudo, constatou-se 
que $60 \%$, de um total de 150 universitários que responderam os questionários, informaram que já utilizaram o metilfenidato durante a vida acadêmica, sendo que $87 \%$ obtiveram o medicamento sem prescrição médica (MOTA; PESSANHA, 2014). No mesmo estudo, os autores observaram uma diferença significativa entre os gêneros dos entrevistados que já haviam utilizado o medicamento, onde $65 \%$ eram do sexo feminino, mas a prevalência de gênero neste trabalho, no entanto, contrasta com outros estudos que não encontraram diferenças significativas entre os gêneros (CALAZANS; BELO, 2017; MORGAN et al., 2017).

A ocorrência de efeitos adversos decorrentes do uso de metilfenidato, entretanto, é comum entre os usuários do medicamento, como demonstrado por diversos autores. Como exemplo, um estudo desenvolvido com 315 estudantes do Centro Universitário Luterano de JiParaná/RO (CEULJI/ULBRA) mostrou que 61,54\% dos estudantes já manifestaram reações adversas relacionadas ao fármaco, o que inclui insônia (34,1\%), dor de cabeça (23,1\%), náusea $(4,4 \%)$, perda de apetite $(5,5 \%)$, palpitação $(17,6 \%)$, boca seca $(13,2 \%)$, entre outros (WILLE, 2018). No estudo já citado e efetuado com alunos dos cursos de Biomedicina, Enfermagem, Farmácia e Nutrição da Faculdade Anhanguera de Brasília, com um total 400 alunos participantes, constatou-se que os efeitos adversos mais frequentes sentidos em função do uso do medicamento, foram dor de cabeça (22\%), taquicardia (22\%), insônia (22\%) e boca seca (I6\%), entre outros que incluem enjoo e dores no corpo (AFFONSO et al., 2016).

\section{CONSIDERAÇÕES FINAIS}

O uso não prescrito do metilfenidato entre estudantes universitários em nosso país é uma realidade que não pode ser ignorada, visto que a utilização indiscriminada deste medicamento, bem como outros psicoestimulantes, pode ocasionar efeitos colaterais imediatos ou a longo prazo, podendo incluir insônia, dependência e psicoses, entre outros. Sendo assim, torna-se necessário que haja um envolvimento de todos os profissionais envolvidos na conscientização dos alunos, bem como no controle da prescrição e dispensação, uma vez que para prescrição deste medicamento é exigida notificação de receita A (cor amarela), de difícil 
acesso, até mesmo para médicos, o que não tem impedido, no entanto, a aquisição pelos usuários.

\section{BIBLIOGRAFIA}

AFFONSO, R. D. S. et al. O uso indiscriminado de psicoestimulantes pelos estudantes da Faculdade Anhanguera de Brasília - Fab. Infarma - Ciências Farmacêuticas, v. 28, n. 3, p. I66I72, 2016.

ANDRADE, L. S. et al. Ritalina uma droga que ameaça a inteligência Ritalina. Revista de Medicina e Saúde de Brasília, v. 7, n. I, p. 99-II2, 2018.

ANVISA. Portaria SVS/MS $\mathrm{n}^{\circ}$ 344, de 12 de maio de 1998. Disponível em: $\langle$ http://antigo.anvisa.gov.br/legislacao\#/visualizar/2629I〉. Acesso em: 2I maio. 202I.

BABCOCK, Q.; BYRNE, T. Student perceptions of methylphenidate abuse at a public liberal arts college. Journal of the American College Health Association, v. 49, n. 3, p. I43-I45, 2000.

BOGLE, K. E.; SMITH, B. H. Illicit methylphenidate use: a review of prevalence, availability, pharmacology, and consequences. Current Drug Abuse Reviews, v. 2, n. 2, p. 157176, 2009.

CALAZANS, A. G. C.; BELO, R. F. C. Prevalência do uso de metilfenidato por estudantes ingressantes nas universidades do município de Sete Lagoas/MG. Revista Brasileira de Ciências da Vida, v. 5, n. I, p. I-26, 2017.

CARNEIRO, S. G. et al. O uso não prescrito de metilfenidato entre acadêmicos de Medicina. Cadernos UniFOA, n. 12, p. 71-8o, 2013.

COLI, A. C. M.; SOUSA E SILVA, M. P.; NAKASU, M. V. P. Uso não Prescrito de Metilfenidato entre Estudantes de uma Faculdade de Medicina do Sul de Minas Gerais. Revista Ciências em Saúde, v. 6, n. 3, p. I2I-132, 2016.

CRF-SP. Brasil registra aumento de $775 \%$ no consumo de Ritalina em dez anos. Disponível em: <http://www.crfsp.org.br/crfsp.org.br/index.php/noticias/5713-consumo-de-ritalinaaumentou-775-em-dez-anos-aponta-pesquisa.html>. Acesso em: 22 out. 2021.

CRUZ, T. C. S. C. et al. Uso não uso não-prescrito de metilfenidato entre estudantes de medicina da Universidade Federal da Bahia. Gazeta Médica da Bahia, v. 8I, n. I, p. 3-6, 201 .

DOMitroviC, N.; CAliman, L. V. As Controvérsias Sócio-Históricas Das Práticas 
Farmacológicas Com O Metilfenidato. Psicologia \& Sociedade, v. 29, n. o, p. I-1o, 2018.

FREESE, L. et al. Non-medical use of methylphenidate: a review. Trends in Psychiatry and Psychotherapy, v. 34, n. 2, p. IIO-II5, 2012.

HARAYAMA, R. et al. Nota Técnica: o consumo de psicofármacos no Brasil, Dados do Sistema Nacional de Gerenciamento de Produtos Controlados Anvisa (2007-2014). Disponível em: $\quad\langle$ http://medicalizacao.org.br/wpcontent/uploads/2015/o6/NotaTecnicaForumnet_v2.pdf >. Acesso em: I4 out. 2021.

ITABORAHY, C.; ORTEGA, F. O metifenidato no Brasil: uma década de publicações. Ciência e Saúde Coletiva, v. I8, n. 3, p. 803-8I6, 2013.

MONTEIRO, B. M. M. et al. Metilfenidato e melhoramento cognitivo em universitários: um estudo de revisão sistemática. SMAD Revista Eletrônica Saúde Mental Álcool e Drogas, v. I3, n. 4, p. 232-242, 2017.

MORGAN, H. L. et al. Consumo de estimulantes cerebrais por estudantes de medicina de uma universidade do extremo sul do Brasil: prevalência, motivação e efeitos. Revista Brasileira de Educação Médica, v. 4I, n. I, p. I02-I09, 2017.

MORTON, W. A.; STOCKTON, G. G. Methylphenidate abuse and psychiatric side effects. Primary Care Companion to the Journal of Clinical Psychiatry, v. 2, n. 5, p. 159-164, 2000.

MOTA, J. S.; PESSANHA, F. F. Prevalência do uso de metilfenidato por universitários de Campos dos Goytacazes, RJ. Revista Vértices, v. I6, n. I, p. 77-86, 2014.

NLM. PubChem Compound Summary for CID 9280, Methylphenidate hydrochloride. Disponível em: <https://pubchem.ncbi.nlm.nih.gov/compound/Methylphenidatehydrochloride>. Acesso em: 21 out. 2021.

ROCHA, K. G. et al. METILFENIDATO: USO INDISCRIMINADO. Revista Saúde dos Vales, v. I, n. I, p. I2-26, 2020.

ROSA, A. C. D. N.; TElleS, M. V. L. TRANSTORNO DE DÉFICIT DE ATENÇÃO E HIPERATIVIDADE EM CRIANÇAS E ADOLESCÊNTES: REVISÃO DE LITERATURA. Id on line Revista de Psicologia, v. 3, n. Io, p. 8I-II7, 2009.

SANTANA, L. C. et al. Consumo de Estimulantes Cerebrais por Estudantes em Instituições de Ensino de Montes Claros/MG. Revista Brasileira de Educação Médica, v. 44, n. I, p. I-8, 2020.

SANTOS, L. DE F.; VASCONCELOS, L. A. Transtorno do déficit de atenção e 
hiperatividade em crianças: Uma revisão interdisciplinar. Psicologia: Teoria e Pesquisa, v. 26, n. 4, p. 717-724, 2010.

WILLE, J. PREVALÊNCIA DO USO DE METILFENIDATO EM ACADÊMICOS DE UM CENTRO UNIVERSITÁRIO EM JI-PARANÁ, RONDÔNIA PREVALENCE. Brazilian Journal of Surgery and Clinical Research-BJSCR, v. 24, n. 3, p. 13-19, 2018. 